\title{
C. elegans fmi-1/flamingo and Wnt pathway components interact genetically to control the anteroposterior neurite growth of the VD GABAergic neurons
}

Brian D Ackley

Department of Molecular Biosciences; University of Kansas; Lawrence, KS USA
Keywords: flamingo, Wnt, axon outgrowth

Submitted: 07/02/13

Accepted: 07/10/13

http://dx.doi.org/10.4161/worm.25715

Correspondence to: Brian D Ackley;

Email: bdackley@ku.edu

Commentary to: Huarcaya Najarro E, Ackley BD. C. elegans fmi-1/flamingo and Wnt pathway components interact genetically to control the anteroposterior neurite growth of the VD GABAergic neurons. Dev Biol 2013; 377:224-35; PMID:23376536; http://dx.doi.org/ 10.1016/j. ydbio.2013.01.014
$\mathrm{D}$ uring development, multiple environmental cues, e.g., growth factors, cell adhesion molecules, etc., interact to influence the pattern of outgrowth of axons and dendrites in a cellspecific fashion. As a result, individual neurons may receive similar signals, but make unique choices, leading to distinct wiring within the nervous system. C. elegans has been useful in identifying molecular cues that influence neuronal development, as well as the downstream mechanisms that allow individual neurons to make cell-specific responses. Recently, we described a role for the conserved cadherin domain-containing protein, FMI-1/flamingo, in multiple stages of neural development in $C$. elegans. During the initial phase of neurite outgrowth, FMI-1 seems to have a relatively cell-specific effect on the VD neurons to promote the initial neurite formed to grow toward the anterior. In this capacity, FMI-1 appears to work coordinately with at least two Wnt ligands, EGL-20 and LIN-44, and multiple downstream Wnt signaling components (including LIN-17/Frizzled, DSH-1/Disheveled, and BAR- $1 / \beta$-catenin). Here I will discuss some of the ideas we considered about how FMI-1 could affect neurons as they acquire their morphology during development.

\section{Elegans Ventral Cord Motor Neurons Exhibit Class-Specific Outgrowth Patterns}

Neural networks are formed when connections are made between neurons and their targets. To find their specific targets, neurons use molecular cues to guide their axons and dendrites to the appropriate fields, and then to match with partners. These cues can be secreted growth factors, extracellular matrix proteins, or cellsurface proteins. Intriguingly, cells can be in relatively similar molecular milieus, yet make different, even opposite reactions, e.g., one cell can be attracted to a molecule, another cell repelled, etc. Such a molecular system was first described in C. elegans, when it was demonstrated that the secreted cue UNC-6/Netrin was necessary for the guidance of both ventrally and dorsally guided axons. ${ }^{1}$ Cells that express UNC-40/DCC as the UNC-6 receptor are attracted to the ventral midline, the UNC-6 source, while those that express both UNC-40 and UNC-5 are repelled from UNC-6. ${ }^{2-4}$

The primary motor neurons in C. elegans form during two stages. First, during embryogenesis, the $\mathrm{DA}, \mathrm{DB}$, and $\mathrm{DD}$ neurons are formed, and then near the end of the first larval stage (L1) the VA, VB, VC, VD, and AS neurons form. With the exception of the DD and VD neurons, which have morphologies that are similar to one another, each of these classes can be distinguished by its distinct pattern of neurite (axon/dendrite) outgrowth (Fig. 1). Because the shape of each neuron is a reflection of its function in the animal, it is worth thinking about how these cells acquire their morphology.

In fmi-1 mutant animals we and others noticed defects primarily occurred in axons along the $\mathrm{A} / \mathrm{P}$ axis, e.g., neurons fail to fully extend to their normal 


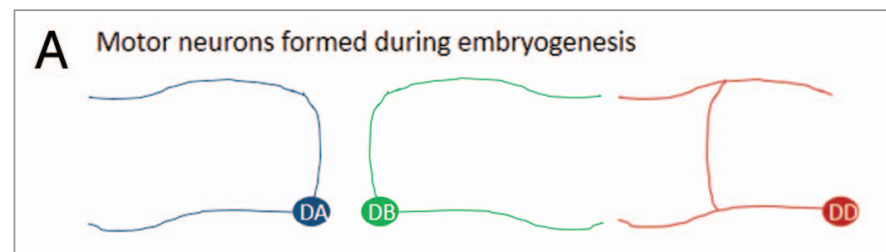

B Motor neurons formed in L1

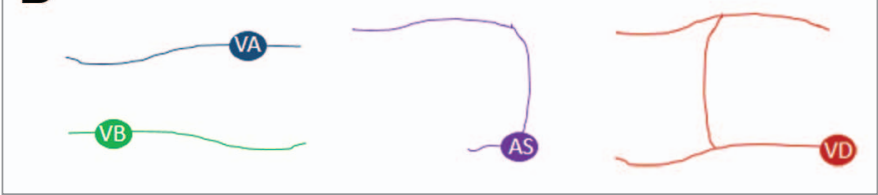

Figure 1. Ventral cord motor neuron morphology by class. (A) Outgrowth patterns of the nine DA and seven DB cholinergic motor neurons and six DD GABAergic motor neurons that form during embryogenesis. (B) And the same for the $12 \mathrm{VA}, 11 \mathrm{VB}, 11$ AS cholinergic motor neurons and $13 \mathrm{VD}$ GABAergic motor neurons that form during the first larval stage. These are the general morphologies, (see http://wormatlas.org/neurons/Individual\%20Neurons/Neuronframeset.html for details on individual neurons). In both ( $\mathbf{A}$ and $\mathbf{B}$ ) the left is anterior and top is dorsal. Some of the motor neurons, in general, extend a single long process from the cell body (AS, DD, VD, VA, VB) and others extend two separate long processes (DA and $D B$ ), but that these are oriented in different directions along the anterior/posterior (A/P) axis. The DD, VD, DA, and VA neurons extend axons anteriorly, while the $\mathrm{DB}$ and $\mathrm{VB}$ extend axons posteriorly, and the $\mathrm{DA}, \mathrm{DB}$, and $\mathrm{AS}$ neurons extend a process dorsally from the cell body, while DD and VD form dorsal processes from the anterior neurite. Note, the hermaphrodite-specific VC motor neurons are not illustrated. anterior-most termination point.-7 We also observed that in a small subset of VD neurons the initial process appeared to be directed posteriorly. 5 The other cells reported to be affected by fmi-1 mutations do not appear to have this specific phenotype, although the HSN neurons have been reported to turn posteriorly at the midline, rather than anteriorly in fmi-1 mutants. ${ }^{7}$ Interestingly, the DD neurons, which have the same morphology as the VDs, do not display a posterior neurite phenotype in an fmi-1 loss of function background. FMI-1 is present when the DD neurons form, and the loss of fmi-1 can affect DD development, but not apparently in the direction of neurite initiation from the cell, suggesting that FMI-1 is not a general cue to guide anteriorly directed neurons, but rather it appears to selective for some neurons, including the VDs.

In $f m i-1$ mutant animals, on a per cell basis, $-5 \%$ of the VD neurons exhibited a posterior neurite. This indicated that FMI-1 was just one of the signals that instructed VDs to extend an axon anteriorly. Using a candidate gene approach we found that the Wnt ligands EGL-20 and LIN-44 also provide a complimentary signal, as loss of these proteins can also cause a posterior neurite defect in VDs. Mutations in Wnt signaling components could enhance the percentage of the defects found when fmi-1 is also mutated. For example, in fmi-1;dsh-1 double mutants $-12 \%$ of the VD neurons extended posterior neurites. These observations would indicate that there are likely yet more factors that are promoting VDs to project anterior neurites.

We wondered whether fmi-1 mutations were altering cell fate. This did not seem to be the case, as three different cell-specific VD markers were still expressed in the correct number of cells. And, in the neurons with posteriorly directed processes, the rest of the outgrowth pattern appeared to be VD-like (commissural splitting in ventral and dorsal cords), rather than adopting the DB or VB-like morphology. Thus, we ruled out cell-fate errors as the underlying cause of the posterior neurite.

The VD neurons form during L1, and in 11/13 cases, an AS neuron is derived from the neuroblast (VD1 and VD13 are sisters to the VA1 and PDB, respectively). In each case the VD neurons are the posterior daughter of the division, and the sister neuron the anterior one. We were unable to find any instances where the VDs were found anterior to their sister neurons, nor did we observe any changes in the plane of division, suggesting that these events are not obviously dependent on fmi-1.

Work from different systems has suggested that the position of the centrosome influences the place where the axon will initiate. ${ }^{8-11}$ While the evidence for appears to be waning due to work in vivo, thanks to the lineage of all neurons being available in $C$. elegans one can ask if the pattern of cell division during development informs the direction a neurite will grow in differentiated neurons. That is, does being the posterior daughter of a neuroblast division correlate with projecting an anterior neurite and vice versa? Of the 70 $\mathrm{A}, \mathrm{B}$, or D type ventral cord motor neurons, 41 are the posterior daughter, and of those, 23 extend anterior processes, while 18 form posteriorly directed neurites. This makes it unlikely that the position of the centrosome, as a function of the plane of division, instructs in which direction neurites will extend.

\section{VD Motor Neurons Form Axons from Laterally Extended Processes}

Most analyses of axon outgrowth occur using fluorescent labels to visualize motor neurons after outgrowth is completed. In doing so it might appear that anteriorly directed axons emerge from the anterior cortex of the cell. A VD-cell marker, Punc$55: \because g f p$, turns on around the time the AS and VD neurons are dividing, permitting us to observe the early events of neurite initiation (Fig. 2). We found that after dividing to give rise to the VD neuron, the first thing that happens is that the cells extend a lamellipodia-like process to contact the major bundle of the ventral nerve cord. From this structure the cell extends neurites anteriorly, using the nerve cord as an apparent substrate for growth.

We found additional evidence for this in an ultrastructural analysis of an animal at L1/L2 lethargus (available at http:// www.wormimage.org/, worm name: L1_ Lethargus). In that data set, the anterior start of VD3 neuron cell body is around section 220. In this image series, it is possible to see the lateral extension into the nerve cord, and to follow the neurite anteriorly. The posterior limit of the neurite in 
the ventral nerve cord appears to coincide with the terminal point of the cell body around section 253 .

In both the wild-type and the fmi-1 mutant animals we examined we found the neurons could begin to extend neurites in both the anterior and posterior direction, along the ventral nerve cord, out of the lamellipodium. In the wild-type animals, when we could observe two processes, the anterior neurite was generally longer than the posterior one. This is consistent with the anterior growth being favored. When we analyzed the fmi-1; dsh-1 double mutants we found that the length of the anterior and posterior neurites were variable, with either the anterior or posterior neurite longer. This was the first evidence of a difference between our wild-type and our mutant animals, suggesting that this may be at least a part of the posterior neurite phenotype, although that remains to be demonstrated directly.

The ventral nerve cord provides a substrate for the axon outgrowth, and likely contains positional cues that will guide the neurite. We found that during the period of development when VD neurons are forming neurites, FMI-1 is present in the major bundle of the ventral nerve cord. However, we did not observe any apparent gradient of FMI-1 that could provide the neurites with an asymmetry defining the anterior from the posterior. This may be below our limits of detection, or modifications of the substrate could be context dependent. We were able to rescue the posterior neurite defect when we replaced FMI-1 with its endogenous promoter, but not when we used selective expression in a subset of FMI-1 cells, suggesting that there is a requirement for FMI-1 broadly to ensure anterior outgrowth of the VD neurite.

A second point that was apparent from our analysis of the VD development was the timing of the commissural projection. The anterior neurite of the VD branches to form the commissure, but the growth of the anteriorly directed ventral neurite is delayed until the commissure is near or at the dorsal cord. That is, the two neurites are not formed at the same time, rather the commissure forms first and then the anterior branch of the ventral neurite extends to the next $\mathrm{VD}$ along the ventral nerve

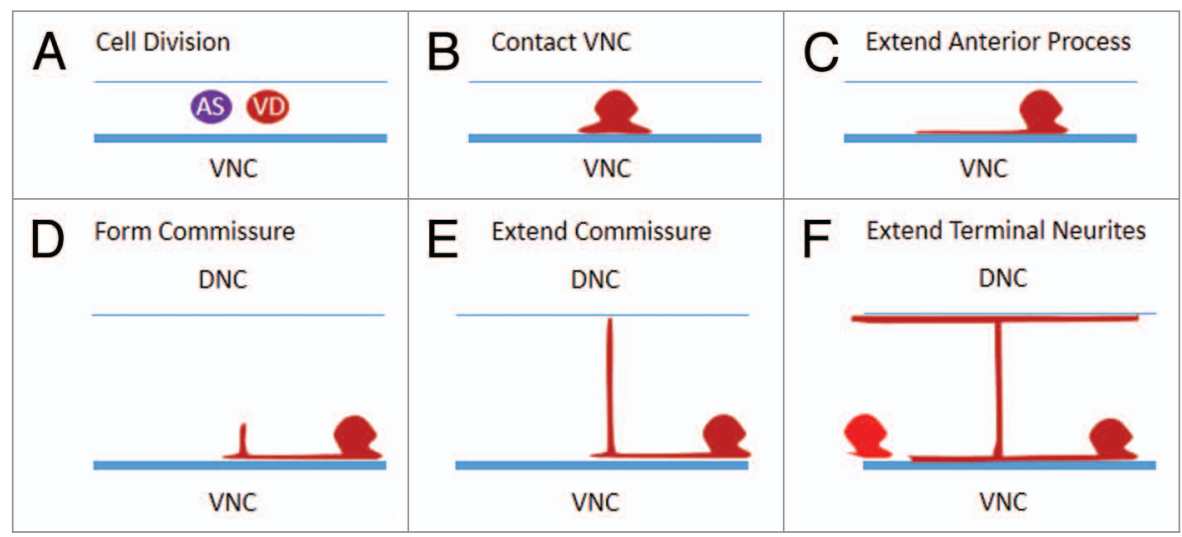

Figure 2. VD motor neuron development. (A) The VD neuron is always the posterior cell formed by the precursor neuroblast. (B) After division the VD neuron extends a lamellipodia-like sheet to contact the ventral nerve cord (VNC). (C) The anterior neurite of the cell grows while the posterior process does not extend. (D) The commissure forms at a point anterior to the cell body, and it grows to reach the dorsal cord $(\mathbf{E})$, before the portion of the neurite anterior to the commissure completes its growth to the next VD anterior (F).

cord (Fig. 2E and F). We were unable to visualize the growth of the dorsal projections because the AS neurites also marked by the Punc-55::gfp obscured their development. Although it is unclear why this would be the case, one might speculate that the guidance machinery within the commissural process would need to be separate from that which will guide the anterior neurite to its termination point. Consistent with that, we found that in fmi-1 mutants the portion of the VD neurite, anterior to the commissure often terminated prior to its expected position, leaving a gap in the ventral nerve cord when visualized with the Punc-55::gfp. Conversely, we observe no cases where a neurite failed to extend dorsally, although we did find a small number of errors in the side of the animal on which it extended. Overall, these data are consistent with FMI-1 functioning as a factor that promotes anterior growth.

Flamingo does function as part of the planar cell polarity (PCP) pathway in Drosophila and vertebrates. We examined other core components of the PCP pathway, including vang-1/Van Gogh, and prkl-1/Prickle, but found no evidence of their involvement in VD directional outgrowth. This is interesting because the Colavita lab has demonstrated that VANG-1 and PRKL-1 proteins are required to repress neurite formation in the VC neurons. ${ }^{12}$ Taken together these data suggest that individual neurons in
C. elegans use distinct cues to acquire their final morphology.

\section{What Polarizes VDs to Promote Anterior Neurite Outgrowth?}

Many questions remain about how FMI-1 is functioning in the VDs to promote anterior outgrowth. Our evidence is consistent with a cell non-autonomous role for FMI-1. Could FMI-1 be functioning in an earlier patterning event, which is subsequently transmitted to the VDs? Our current evidence suggests not. We have examined mutations in genes that can cause phenotypes in pioneer neuron guidance that are similar to those in $\mathrm{fmi}^{-}$ 1 , but these do not recapitulate the posterior neurite phenotype (EHN and BDA, unpublished observations). Although, it may be necessary to test that more rigorously in sensitized backgrounds.

The second explanation is that there is a receptor on the VDs that uses FMI-1 to orient it to the A/P axis. Our previous work suggested that $\mathrm{CDH}-4$, a Fat-like cadherin, was a candidate receptor for FMI-1 with respect to its role in regulating synaptogenesis in the VD neurons, ${ }^{6}$ but $\mathrm{CDH}-4$ does not appear to be contributing to A/P outgrowth.

How might the FMI-1 signal to the VDs manifested as anterior outgrowth of the neurites? What is the machinery that drives the growth cone? Is it the same as that which drives axons along 
the dorsal/ventral axis downstream of UNC-5/UNC-40 signaling? To begin to get a handle on these types of questions we are undertaking a molecular genetic approach. Because of the synergistic increase in phenotypes observed in the fmi-1;dsh-1 double mutants, we are using $d s h-1$ as a sensitized background to conduct genetic screens. We hope in the near future we can have a better understanding of the mechanics of anterior neurite outgrowth and/or neurite initiation.

\section{Disclosure of Potential Conflicts of Interest}

No potential conflicts of interest were disclosed.

\section{Acknowledgments}

BDA was supported in part by funds from The University of Kansas, P20 RR016475 from the INBRE Program of the NCRR, and RC1 GM091086 from the NIH. The work described was conducted primarily by Elvis Huarcaya Najarro (EHN). I thank members of the Ackley lab for their critical reading of this manuscript.

\section{References}

1. Hedgecock EM, Culotti JG, Hall DH. The unc5 , unc- 6 , and unc- 40 genes guide circumferential migrations of pioneer axons and mesodermal cells on the epidermis in C. elegans. Neuron 1990; 4:61-85, PMID:2310575; http://dx.doi.org/10.1016/08966273(90)90444-K

2. Ishii N, Wadsworth WG, Stern BD, Culotti JG, Hedgecock EM. UNC-6, a laminin-related protein, guides cell and pioneer axon migrations in C. elegans. Neuron 1992; 9:873-81; PMID:1329863; http:// dx.doi.org/10.1016/0896-6273(92)90240-E

3. Hamelin M, Zhou Y, Su MW, Scott IM, Culotti JG. Expression of the UNC-5 guidance receptor in the touch neurons of $\mathrm{C}$. elegans steers their axon dorsally. Nature 1993; 364:327-30; PMID:8332188; http://dx.doi.org/10.1038/364327a0

4. Chan SS, Zheng H, Su MW, Wilk R, Killeen MT, Hedgecock EM, et al. UNC-40, a C. elegans homolog of DCC (Deleted in Colorectal Cancer), is required in motile cells responding to UNC-6 netrin cues. Cell 1996; 87:187-95; PMID:8861903; http:// dx.doi.org/10.1016/S0092-8674(00)81337-9

5. Huarcaya Najarro E, Ackley BD. C. elegans fmi-1/ flamingo and Wnt pathway components interact genetically to control the anteroposterior neurite growth of the VD GABAergic neurons. Dev Biol 2013; 377:224-35; PMID:23376536; http://dx.doi. org/10.1016/j.ydbio.2013.01.014

6. Najarro EH, Wong L, Zhen M, Carpio EP, Goncharov A, Garriga G, et al. Caenorhabditis elegans flamingo cadherin fmi-1 regulates GABAergic neuronal development. J Neurosci 2012; 32:4196211; PMID:22442082; http://dx.doi.org/10.1523/ JNEUROSCI.3094-11.2012
7. Steimel A, Wong L, Najarro EH, Ackley BD, Garriga G, Hutter H. The Flamingo ortholog FMI-1 controls pioneer-dependent navigation of follower axons in C. elegans. Development 2010; 137:3663-73; PMID:20876647; http://dx.doi.org/10.1242/ dev.054320

8. Kuijpers M, Hoogenraad CC. Centrosomes, microtubules and neuronal development. Mol Cell Neurosci 2011; 48:349-58; PMID:21722732; http://dx.doi. org/10.1016/j.mcn.2011.05.004

9. Stiess M, Maghelli N, Kapitein LC, Gomis-Rüth $\mathrm{S}$, Wilsch-Bräuninger $\mathrm{M}$, Hoogenraad $\mathrm{CC}$, et al. Axon extension occurs independently of centrosomal microtubule nucleation. Science 2010; 327:704-7; PMID:20056854; http://dx.doi.org/10.1126/science.1182179

10. Distel M, Hocking JC, Volkmann K, Köster RW. The centrosome neither persistently leads migration nor determines the site of axonogenesis in migrating neurons in vivo. J Cell Biol 2010; 191:875 90; PMID:21059852; http://dx.doi.org/10.1083/ jcb.201004154

11. de Anda FC, Pollarolo G, Da Silva JS, Camoletto PG, Feiguin F, Dotti CG. Centrosome localization determines neuronal polarity. Nature 2005; 436:704 8; PMID:16079847; http://dx.doi.org/10.1038/ nature 03811

12. Sanchez-Alvarez L, Visanuvimol J, McEwan A, Su A, Imai JH, Colavita A. VANG-1 and PRKL1 cooperate to negatively regulate neurite formation in Caenorhabditis elegans. PLoS Genet 2011 7:e1002257; PMID:21912529; http://dx.doi. org/10.1371/journal.pgen.1002257 